\title{
OPEN A surfactant polymer wound dressing protects human keratinocytes from inducible necroptosis
}

\begin{abstract}
Puneet Khandelwal ${ }^{1,3}$, Amitava Das ${ }^{1,3}$, Chandan K. Sen ${ }^{1}$, Sangly P. Srinivas ${ }^{2}$, Sashwati Roy ${ }^{1}$ \& Savita Khanna ${ }^{1 \bowtie}$

Chronic wounds show necroptosis from which keratinocytes must be protected to enable appropriate wound re-epithelialization and closure. Poloxamers, a class of synthetic triblock copolymers, are known to be effective against plasma membrane damage (PMD). The purpose of this study is to evaluate the efficacy of a specific poloxamer, surfactant polymer dressing (SPD), which is currently used clinically as wound care dressing, against PMD in keratinocytes. Triton X-100 (TX100) at sub-lytic concentrations caused PMD as demonstrated by the efflux of calcein and by the influx of propidium iodide and FM1-43. TX100, an inducer of necroptosis, led to mitochondrial fragmentation, depletion of nuclear HMGB1, and activation of signaling complex associated with necroptosis (i.e., activation of RIP3 and phosphorylation of MLKL). All responses following exposure of human keratinocytes to TX100 were attenuated by pre- or co-treatment with SPD $(100 \mathrm{mg} / \mathrm{ml})$. The activation and translocation of phospho-MLKL to the plasma membrane, taken together with depletion of nuclear HMGB1, characterized the observed cell death as necroptosis. Thus, our findings show that TX100induced plasma membrane damage and death by necroptosis were both attenuated by SPD, allowing keratinocyte survival. The significance of such protective effects of SPD on keratinocytes in wound re-epithelialization and closure warrant further studies.
\end{abstract}

The chronic wound microenvironment is harsh often characterized by un-resolved inflammation, elevated protease activity, low $\mathrm{pH}$, high peroxide levels and large masses of dead and dying cells ${ }^{1-4}$. Surgical debridement and negative pressure wound therapy are commonly used to cleanse the wound microenvironment ${ }^{5-7}$. After appropriate wound bed preparation ${ }^{8}$, wound dressings are intended to maintain favorable changes in the wound microenvironment such that the body's healing responses may be effective in closing the wound ${ }^{9-11}$. In a wound defect on the skin, keratinocytes are the primary cells that achieve wound closure by re-epithelialization ${ }^{12}$. Thus, keratinocyte survival and migration are critically required to achieve this primary endpoint of wound healing. Wound dressings favoring keratinocyte survival in a hostile wound microenvironment are therefore of extraordinary significance.

At the open chronic wound-site, death of skin cells may be caused by a multitude of chemical mediators and mechanical injury. The chemical mediators in the form of pore-forming agents released by microbes directly compromise the integrity of the plasma membrane, potentially causing electrolyte imbalance and inflammasome activation ${ }^{13-22}$. On the other hand, chemical mediators in the form of reactive oxygen species, DAMPs (damage-associated molecular patterns), and cytokines emerging from the activity of pro-inflammatory cells breach plasma membrane integrity ${ }^{17,23-29}$. Such an insult is known to cause necroptosis (a programmed form of necrosis) in the chronic wound ${ }^{27,30}$. Previously, necrosis was considered as uncontrolled or accidental cell death caused by physical or chemical trauma. However, current evidence demonstrates necroptosis is regulated by intrinsic cellular proteins such as receptor interacting protein 3 (RIP3) and mixed lineage kinase domain-like (MLKL). Necroptosis is characterized by swelling and coalescing of intracellular organelles, loss of cellular ATP content, rupture of the plasma membrane, and subsequent leakage of cell contents ${ }^{31,32}$.

${ }^{1}$ Department of Surgery, Indiana Center for Regenerative Medicine and Engineering, Indiana University School of Medicine, 975 W Walnut St, Medical Research Library Building, Indianapolis, IN 46202, USA. 2School of Optometry, Indiana University, 800 East Atwater Avenue, Bloomington, IN 47405, USA. ${ }^{3}$ These authors contributed equally: Puneet Khandelwal and Amitava Das. ${ }^{\varpi}$ email: sjkhanna@iu.edu 
Non-ionic synthetic triblock copolymers, also known as poloxamers, are cognized for their function as membrane sealant ${ }^{33,34}$. Poloxamers have been evaluated against PMD in different pathological states, including respiratory dysfunctions, muscular dystrophy, sickle cell anemia, and neurodegenerative diseases such as Alzheimer's disease ${ }^{33}$. A particular triblock copolymer, Poloxamer 188, is the principal component of a wound dressing, viz., surfactant polymer dressing (SPD) ${ }^{35}$. Polymicrobial biofilm infection impairs functional wound closure as evident by deficient restoration of skin barrier function at the wound-site ${ }^{36,37}$. The anti-biofilm properties of SPD are, therefore, of value as wound care dressing ${ }^{35}$.

Complicated wounds are known to necrotic tissue burden which are clinically removed by surgical debridement and other such processes ${ }^{36,38}$. During such removal of dead tissue, it becomes important for a dressing like SPD to provide support to injured cells in a way that favors tissue repair. The site of injury is populated by a large number of skin cells that suffer from membrane damage secondary to infection and inflammation ${ }^{39-43}$. The effects of SPD on host skin cells remain unclear, however. In the experimental system studied, it has been our objective to test the effectiveness of SPD against PMD in keratinocytes to support membrane repair of injury affected cells such that they may participate in the overall process of skin repair. Triton X-100 (TX100) was recognized and utilized as a potent inducer of keratinocyte necroptosis at a sub-lytic dose employed.

\section{Results}

Triton X-100 induces cell death. Cell death was assessed in human keratinocytes (HaCaT) by following the influx of PI and efflux of calcein as measured by flow cytometry and confocal microscopy. An increase in uptake of PI and efflux of calcein are markers of cell death. Exposure to 0.1, 0.2, and $0.3 \mathrm{mM}$ of TX100 for $3 \mathrm{~h}$ led to $\sim 4 \%, \sim 60 \%$, and $\sim 85 \%$, respectively (Fig. $1 \mathrm{~A}, \mathrm{~B}$ ) as measured by flow cytometry. The confocal images (Fig. 1C,D) supports the flow cytometry data. For further experiments, $0.2 \mathrm{mM}$ of TX100 was chosen since this dose was sublytic and below the critical micellar concentration (CMC) of TX100. TX100-induced cell death was evident at $3 \mathrm{~h}$, as observed by flow cytometry (Fig. 1E,F). Time-dependent exposure (3, 6, and $9 \mathrm{~h}$ ) of TX100 did not increase the cell death (Fig. 1E,F).

Triton X-100 induced cell death was blocked by pre- and co-treatment with SPD. Exposure to SPD alone was not toxic to cells at concentrations of up to $100 \mathrm{mg} / \mathrm{ml}$ (Fig. S1). To assess the efficacy of SPD against TX100-induced cell death, HaCaT cells were pre-treated with SPD at different concentrations (20,50, and $100 \mathrm{mg} / \mathrm{ml}$ ) for $24 \mathrm{~h}$ and then exposed cells to TX100 in the continued presence of SPD. Data from flow cytometry (Fig. 2A,B) and confocal microscopy (Fig. 2C,D) demonstrate that SPD consistently reduced cell death in response to $3 \mathrm{~h}$ exposure to TX100 at $0.2 \mathrm{mM}$. Similar findings were observed in human epidermal keratinocytes (HEKa) cells, where pre-treatment with SPD at a concentration of $100 \mathrm{mg} / \mathrm{ml}$ was able to reduce TX100-induced cell death (Fig. S2). In addition, HaCaT cells were co-treated with TX100 and SPD simultaneously (without pretreatment with SPD), and cell death was analyzed by flow cytometry and confocal microscopy. Co-treatment with SPD was effective against TX100-induced cell death (Fig. 2E-H). To test if the protective efficacy of SPD over TX100-induced cell death persisted long-term, HaCaT cells were co-treated with TX100 and SPD (100 mg/ $\mathrm{ml}$ ) for $3 \mathrm{~h}$, washed off, and then subjected to analysis of cell death after $24 \mathrm{~h}$. Interestingly, TX100-induced cell death was significantly less when co-treated with SPD (Fig. 3A-D).

SPD maintained the integrity of the plasma membrane following exposure to Triton $\mathrm{X}-100$. Exposure to TX100 resulted in intense staining in the cytoplasm, indicating movement of the dye (FM1-43) and subsequent intercalation into membranes of the cytoplasmic organelles (Fig. 4A,B). The accumulation was significantly attenuated in the presence of SPD (Fig. 4A,B).

SPD reduced nuclear depletion of HMGB1 and mitochondrial fragmentation. High mobility group protein B1 (HMGB1) is a nuclear protein vital for the maintenance of several nuclear functions, including replication, recombination, transcription, and DNA repair ${ }^{44,45}$. Nuclear depletion of HMGB1 is a marker of necroptosis ${ }^{46}$. We sought to assess the nuclear abundance of HMGB1 post-insult. Immunocytochemistry with an antibody specific to HMGB1 was performed to assess its cellular localization. Nuclear depletion of HMGB1, a classical marker of necroptosis, was observed in response to TX100 (Fig. 5A-E, S3). In agreement with protection against cell death, SPD at $100 \mathrm{mg} / \mathrm{ml}$ prevented the nuclear depletion of HMGB1 (Fig. 5A-E, S3). Yet another important role ascribed to HMGB1 is in mitochondrial quality control ${ }^{47}$. Thus, we examined if the mitochondria are influenced concomitant with HMGB1 depletion. For this purpose, cells were treated with SPD and TX-100 for $1 \mathrm{~h}$, followed by staining with MitoTrackerRedCMXRos. Exposure to TX100 at $0.2 \mathrm{mM}$ led to a fragmentation of the tubular mitochondria (Fig. 6A,B). The reduced length of mitochondria in response to TX100 was blocked by co-treatment with SPD (Fig. 6A,B).

Triton X-100 induced upregulation of RIP3 kinase as well as activation of MLKL. Among the patterns of cell death in response to TX100, an involvement of apoptosis, including the canonical pathways, has been questioned ${ }^{48-50}$. Since there is a potential for the release of DAMP (such as HMGB1), we examined programmed necrosis, i.e., necroptosis, following treatment with TX100. Here, we focused on the signaling complex upstream of necroptosis. As a first step, we tested RIP3 expression by immunocytochemistry after TX100 treatment. Exposure to TX100 for $3 \mathrm{~h}$ led to an increase of RIP3, which was attenuated by the co-treatment with SPD (Fig. 7A,B). To test the role of MLKL, we performed immunocytochemistry of phospho-MLKL. MLKL was found to be phosphorylated upon treatment with TX100, which was significantly attenuated by co-treatment with SPD (Fig. 8A,B). 
A
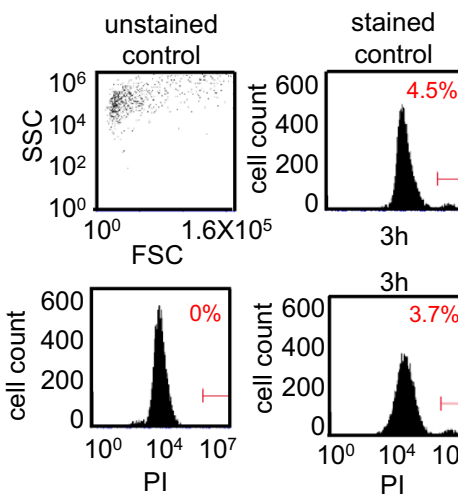

unstained control

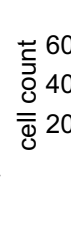

3h



TX100

(0.1mM)
TX100

$(0.2 \mathrm{mM})$

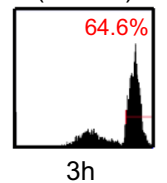

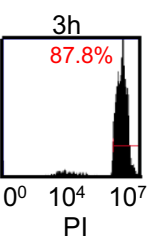

TX100

$(0.3 \mathrm{mM})$

C

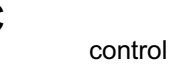
TX100 $(0.1 \mathrm{mM})$

Oh

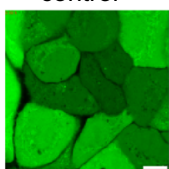

$3 \mathrm{~h}$
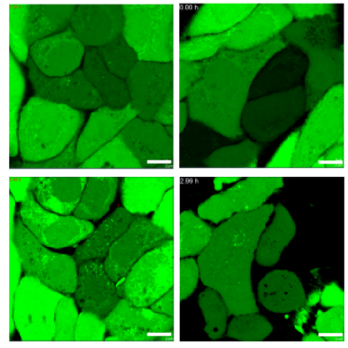

TX100

$(0.2 \mathrm{mM})$

TX100

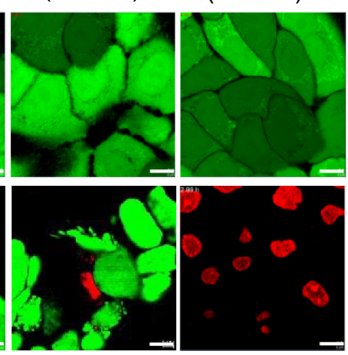

E


nstaine

control

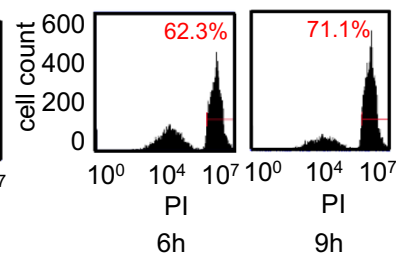

$6 \mathrm{~h}$

9h
B

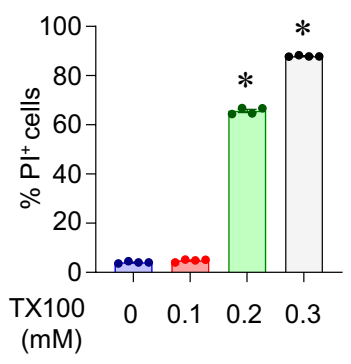

D

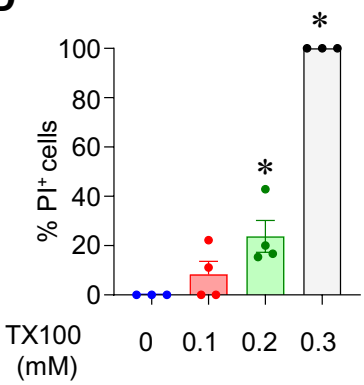

F

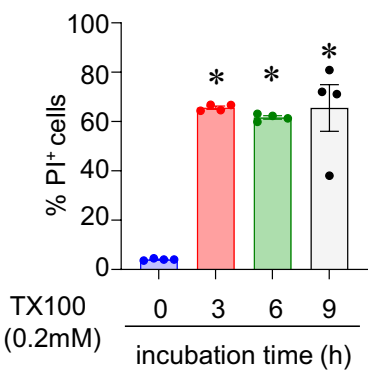

Figure 1. TX100-induced cell death is dose-dependent. Human keratinocytes (HaCaT) were exposed to TX100 at different concentrations and durations. Cells were stained with PI for flow cytometry and Calcein and PI for confocal microscopy. (A) Flow cytometry histograms showing \%PI-positive cells upon treatment with increasing concentration ( $0.1 \mathrm{mM}, 0.2 \mathrm{mM}$ and $0.3 \mathrm{mM}$ ) of TX100 for $3 \mathrm{~h}$. (B) Quantification of \%PI-positive cells from flow cytometry representing mean $\pm \operatorname{SEM}(n=4)$. $(\mathbf{C}, \mathbf{D})$ Representative confocal images and quantification of \%PI-positive cells after $3 \mathrm{~h}$ exposure to increasing levels of TX100. Scale, $10 \mu \mathrm{m}$. Data represent mean \pm SEM $(n=3-4)$. (E,F) Flow cytometry measurements (\%PI-positive cells) of HaCaT keratinocytes exposed to TX100 $(0.2 \mathrm{mM})$ for 3,6 , and $9 \mathrm{~h}$. Data represent mean $\pm \operatorname{SEM}(\mathrm{n}=4) .{ }^{*} p<0.05$ compared to control (TX100-untreated).

\section{Discussion}

SPD is employed as a water-soluble burn wound dressing and is FDA cleared ${ }^{51,52}$. SPD is not only well tolerated by patients, but anecdotal clinical evidence show beneficial effects in wound healing ${ }^{53,54}$. It is therefore of extraordinary significance to understand the mechanism of action of SPD. Having a hydrophilic polymer chain, SPD can form a reservoir for topical hydrophilic antimicrobials agents ${ }^{55}$. SPD can be removed from wound beds easily compared to standard silver sulfadiazine creams on the burn wounds. They can effectively disrupt bacterial biofilm infection ${ }^{35}$. Despite several advantages, cellular mechanisms contributing to the efficacy of SPDs on cytoprotection under conditions inciting PMD remain yet to be fully resolved. During PMD, necroptosis 
Figure 2. Pre- and co-treatment of human keratinocytes with SPD protected against TX100-induced cell death. (A-D) Human keratinocytes (HaCaT) were pretreated with SPD $(100 \mathrm{mg} / \mathrm{ml})$ for $24 \mathrm{~h}$ and then exposed to TX100 $(0.2 \mathrm{mM})$ for $3 \mathrm{~h}$ in the continued presence of SPD $(100 \mathrm{mg} / \mathrm{ml})$. (A,B) Cells were stained with PI for flow cytometry. Flow cytometry histograms and quantification of \%PI-positive cells. Data represent mean \pm SEM $(\mathrm{n}=4) .{ }^{*} p<0.05$ compared to control (TX100-untreated). ${ }^{\dagger} p<0.05$ compared to TX100-treated group. (C,D) Cells were stained with Calcein and PI for confocal microscopy. Representative images and quantification of $\%$ PI-positive cells are shown. Data represent mean \pm SEM $(n=3-4) .{ }^{*} p<0.05$ compared to control (TX100untreated). ${ }^{\dagger} p<0.05$ compared to TX100-treated group. (E-H) Human keratinocytes (HaCaT) were co-treated with SPD $(100 \mathrm{mg} / \mathrm{ml})$ and TX100 $(0.2 \mathrm{mM})$ for $3 \mathrm{~h}$. (E,F) Cells were stained with PI for flow cytometry. Flow cytometry histograms and quantification of \%PI-positive cells. Data represent mean \pm SEM $(n=4) .{ }^{*} p<0.05$ compared to control (TX100-untreated). ${ }^{\dagger} p<0.05$ compared to TX100-treated group. $(\mathbf{G}, \mathbf{H})$ Cells were stained with Calcein and PI for confocal microscopy. Representative images and quantification of \%PI-positive cells are shown. Scale, $10 \mu \mathrm{m}$. Data represent mean \pm SEM $(\mathrm{n}=3-4) .{ }^{\star} p<0.05$ compared to control (TX100-untreated). ${ }^{\dagger} p<0.05$ compared to TX100-treated group.

form of cell death is evident at the site of chronic wounds ${ }^{56}$. The present work characterizes TX100 as a potent inducer of necroptosis in human keratinocytes. At dosages achievable at the wound site, where SPD is delivered topically, SPD prevented necroptosis induced by TX100. Any sparing of keratinocyte death at the hostile wound microenvironment is likely to facilitate wound-re-epithelialization and therefore closure.

As strategies to induce reproducible PMD, many approaches have been reported ${ }^{57-62}$. They include (a) laserinduced membrane damage, (b) exposure to pore-forming pathogenic cytotoxins, (c) mechanical injury by glass beads, shear stress, cellular contraction, cell scraping, and forcing cells through a needle, and (d) exposure to detergents ${ }^{63,64}$. Of these methods, the pore-forming toxins and detergents are suitable for induction of PMD simultaneously in a large number of cells ${ }^{63}$, and hence are highly suitable for high-throughput screening of potential therapeutic agents. However, in the case of bacterial cytotoxins, a significant disadvantage is several toxins would be required to produce membrane pores of varying sizes. With detergents, on the other hand, the extent of PMD can be tuned by varying their concentration and time of exposure. These variables can easily be controlled during the conduct of experiments. Controlled exposure to TX100, a non-ionic detergent, is widely employed in cell biological applications $\mathrm{s}^{50,65,66}$. The interactions of TX100 with the plasma membrane in other cell types have been partly characterized. In particular, at sub-lytic doses, TX100 is known to permeabilize the plasma membrane and cause cell death by mechanisms not yet established ${ }^{48-50,65-67}$. The sub-lytic dose, however, corresponds to levels below CMC, which is $\sim 0.24 \mathrm{mM}^{48}$. Because of their polar head group, TX100 molecules disrupt hydrogen bonding present within the cell's lipid bilayer and lead to the destruction of the compactness and integrity of lipid membrane ${ }^{65}$. Cells undergo irreversible permeabilization of the membrane and structural collapse when the TX100 concentration reached the CMC ${ }^{65}$. The action of TX100 also depends on the composition of the lipid bilayer ${ }^{68}$. TX100 action starts by destabilization of the lipid component of the membranes (noncooperative binding of detergents to the membrane to the cooperative binding). This leads to the formation of membrane fragments of proteins and lipids with detergent shielded edges ${ }^{69}$. The changes in plasma membrane permeability and fluidity also plays a critical role in psoriasis ${ }^{70}$.

The non-ionic detergent, TX100, is known to elicit plasma membrane damage (demonstrated usually by transiently increased membrane permeability) and subsequent cell death ${ }^{48-50,66,67,71,72}$. In some cells, TX100 induces apoptotic cell death ${ }^{48-50,66,67}$. Lipid homeostasis of the skin is unique such that lipid signaling is a key contributor to the turn-over of skin cells and the lipid composition of the skin contributes to its barrier function ${ }^{73}$. It is therefore not surprising that keratinocytes respond differently to detergents. For example, NP-40 is a non-ionic detergent like TX100 yet it induces necrosis in HaCaT keratinocytes ${ }^{74}$. Our choice to make use of TX100 to induce experimental PMD turned out to be a useful strategy to produce damage in a large number of cells simultaneously. At sub-lytic concentrations, TX100 produced transient membrane permeabilization without causing immediate cell death. Such membrane permeabilization is known to be a characteristic feature of necroptosis ${ }^{29}$. SPD, by itself, showed no cytotoxicity. When co-treated to cells and subsequently removed, SPD retained its cytoprotective properties indicating of successful cellular uptake by a magnitude that was functional relevant. Cytoprotective effects of SPD co-treated with TX100 demonstrated that SPD is not only safe on its own but that it did not chemically interact with TX100 in a harmful manner.

FM1-43 is a fluorescent membrane probe ${ }^{75}$. It is not fluorescent in an aqueous medium. However, the dye becomes intensely fluorescent when it intercalates into the outer leaflet of the plasma membrane ${ }^{75}$. The fluorescence of FM1-43 is only at the membrane. An increase in fluorescence is an indicator of plasma membrane damage $^{76}$. In keratinocytes, TX100 dependent increase in FM1-43 fluorescence establishes the ability of the detergent to cause PMD. PMD is known to occur during necroptosis ${ }^{29}$. This work is the first to recognize the value of FM1-43 as a productive approach to detect early necroptosis events. Understanding of mitochondrial changes during the course of necroptosis has led to the observation that the RIP1-RIP3 complex initiates mitochondrial fission ${ }^{77}$. Co-ordinated with DNA replication, mitochondrial fission divides the organelle. In a healthy cell, mitochondria exist as a dynamic network of fission and fusion ${ }^{78}$. During necroptosis, the balance favors fission and manifests as reduced mitochondrial length ${ }^{79}$. MitoTrackerRedCMXRos is a cationic dye, which accumulates in the mitochondrial membrane of living cells ${ }^{80}$. It is intrinsically fluorescent, binds irreversibly to the polarized mitochondrial membrane, and does not require reduction or oxidation for the emission of fluorescence ${ }^{80}$. Thus, it is well suited to study mitochondrial fission during necroptosis. TX100 dependent reduction in mitochondrial length, indicative of fission, was completely spared in the presence of SPD. 


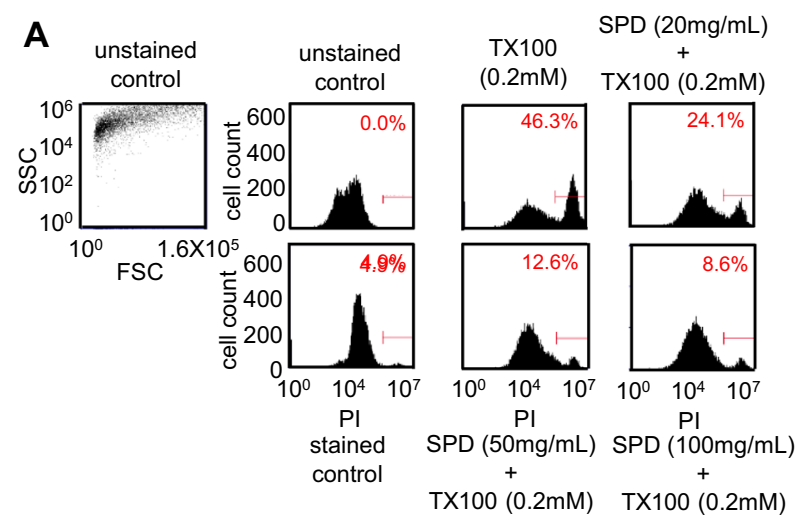

B

C

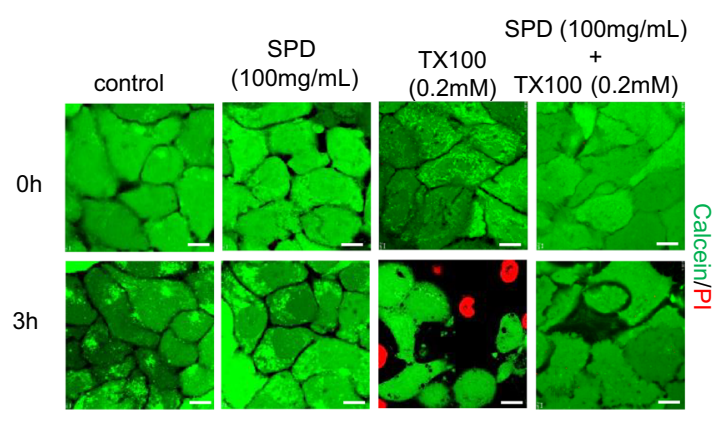

D
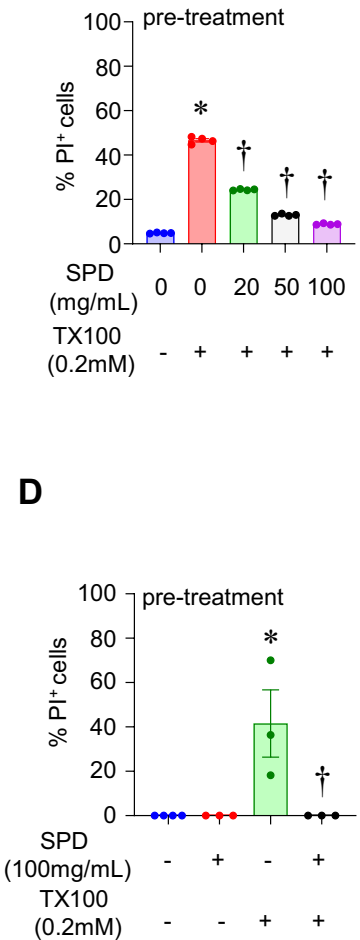

E

SPD $(20 \mathrm{mg} / \mathrm{mL})$

$\mathbf{F}$
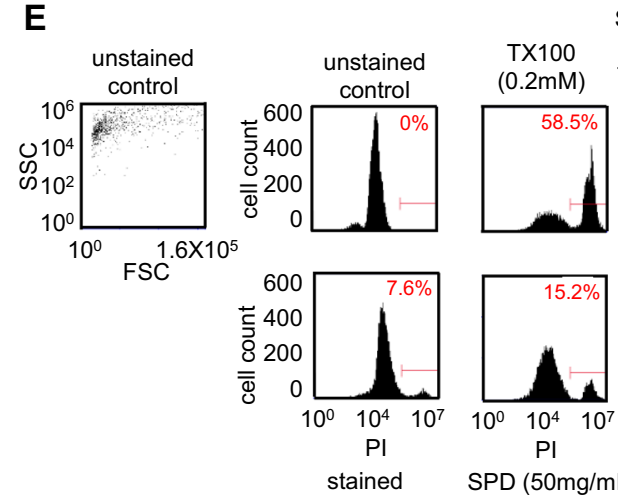

X100 (0.2mM)

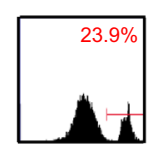

control

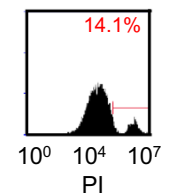

SPD $(50 \mathrm{mg} / \mathrm{mL})$ SPD $(100 \mathrm{mg} / \mathrm{mL})$

$+$

TX100 (0.2mM) TX100 (0.2mM)

G

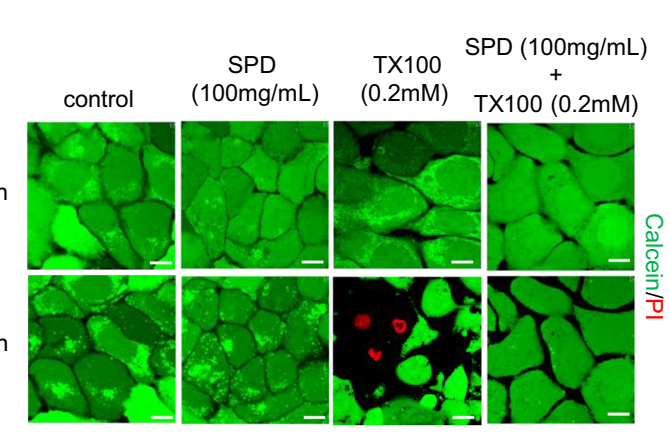

H
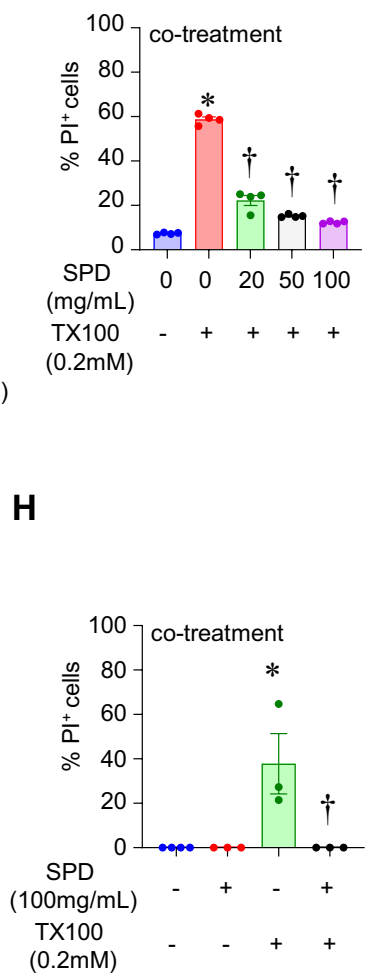


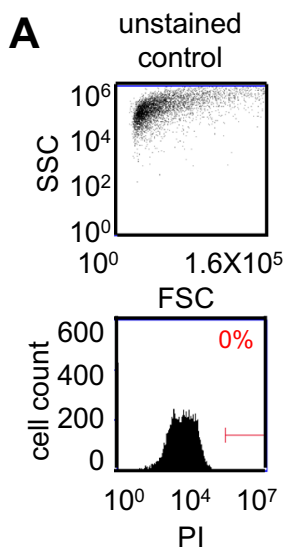

C

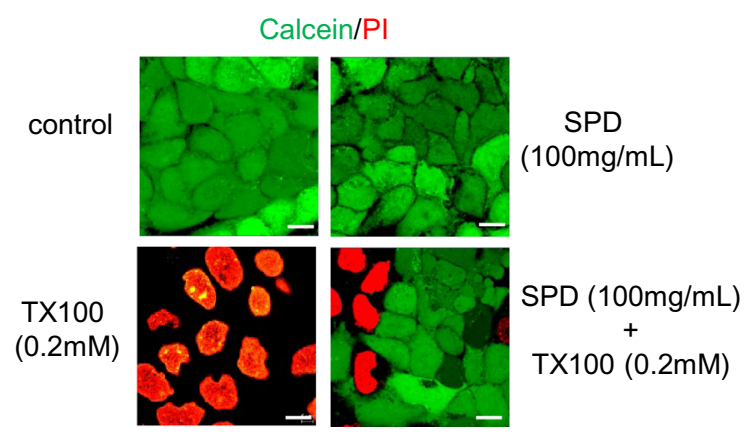

TX100

$(0.2 \mathrm{mM})$
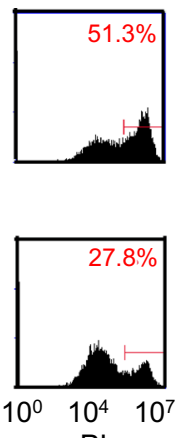

$\mathrm{PI}$



$(100 \mathrm{mg} / \mathrm{mL})$
TX100 (0.2mM)

SPD $(100 \mathrm{mg} / \mathrm{mL})$

B
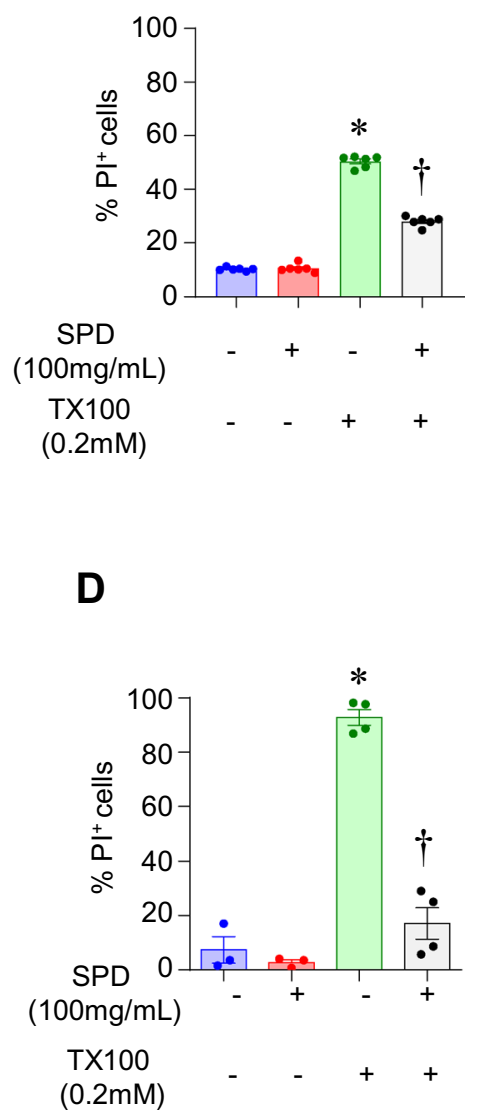

Figure 3. Prolonged efficacy of SPD against TX100-induced cell death. Human keratinocytes (HaCaT) were first co-treated with SPD $(100 \mathrm{mg} / \mathrm{ml})$ and TX100 $(0.2 \mathrm{mM})$ for $3 \mathrm{~h}$. Cells were subsequently washed with PBS and incubated in a fresh medium for $24 \mathrm{~h}$ containing SPD and then analyzed for cell death by flow cytometry and confocal microscopy. (A,B) Flow cytometry histograms and quantification of \%PI-positive cells. Data represent mean $\pm \operatorname{SEM}(\mathrm{n}=6) .{ }^{*} p<0.05$ compared to control (TX100-untreated). ${ }^{\dagger} p<0.05$ compared to TX100treated group. (C,D) Confocal microscopy images and quantification of \%PI-positive cells. Scale, $10 \mu \mathrm{m}$. Data represent mean \pm SEM $(n=3-4) .{ }^{*} p<0.05$ compared to control (TX100-untreated). ${ }^{\dagger} p<0.05$ compared to TX100-treated group.

HMGB1 is a ubiquitous nuclear protein, the release of which is a characteristic feature of necroptosis ${ }^{46}$. Nuclear HMGB1 is not only released as a DAMP but is also associated with mitochondrial quality control ${ }^{47}$. Loss of nuclear HMGB1 is known to cause mitochondrial fission ${ }^{81,82}$. In the nucleus, the cell-survival functions of HMGB1 are attributed to its ability to facilitate assembly of DNA binding proteins on chromatin ${ }^{83}$. Once released from the nucleus, HMGB1 interacts with specific TLRs and incites local inflammation ${ }^{84-86}$. Thus, the release of nuclear HMGB1 is counterproductive to the wound healing cascade where timely resolution of inflammation is critical ${ }^{2}$. TX100 caused rapid and overt depletion of nuclear HMGB1. When expressed as the ratio of nuclear:extranuclear HMGB1, TX100 caused marked depletion which was significantly rescued under conditions of SPD co-treatment.

The canonical pathway for the induction of necroptosis entails activation of RIP1K-RIP3K, followed by activation of phospho-MLKL. Activation of RIP1K, a serine-threonine kinase causes oligomerization and autophosphorylation of RIP3K at Ser227 ${ }^{87-90}$. RIP3K, thus activated, drives phosphorylation of MLKL ${ }^{28,87,91,92}$. The phosphorylated MLKL is translocated to the plasma membrane leading to pore formation reminiscent of gasdermins in pyroptosis ${ }^{28,87,91,92}$. As a pseudokinase, MLKL does not target any protein for phosphorylation. Instead, upon activation, MLKL is trafficked to the plasma membrane 8, $^{28,87,93}$. Upon interaction with inositide lipids associated with the plasma membrane, MLKL induces necroptosis causing cell death ${ }^{91}$. In particular, MLKL at the plasma membrane form pores that are directly implicated in cell death. In this work, TX100 activated RIP3 and generated phospho-MLKL. Moreover, in agreement with the spared depletion of nuclear HMGB1, the abundance of both RIP3 and phospho-MLKL were blunted in the presence of SPD. Inhibition of keratinocyte necroptosis is known to be a protective effect against psoriatic inflammation ${ }^{94}$. The clinical relevance of SPD may thus be broader than the realm of chronic wound management.

Taken together, this work shows that SPD, clinically used as wound care dressing, is likely to have functions beyond its reported effects on biofilm management. Necroptotic death, known to be abundant in chronic wounds, can be managed by SPD. The effects of this poloxamer are evident in multiple signaling events leading 



Figure 4. SPD protects against TX100-induced plasma membrane damage. Human keratinocytes (HaCaT) were exposed to TX100 $(0.2 \mathrm{mM})$ for $3 \mathrm{~h}$ in presence of SPD $(100 \mathrm{mg} / \mathrm{ml})$. Cells were stained with FM1-43 and subjected to microscopy. (A,B) Representative microscopic images and quantification of intensity of FM1-43 was assessed by confocal microscopy. Scale, $5 \mu \mathrm{m}$. Data represent mean \pm SEM $(n=4-6){ }^{*} p<0.05$ compared to control (TX100-untreated). ${ }^{\dagger} p<0.05$ compared to TX100-treated group.

to necroptosis. Sparing of keratinocyte nuclear HMGB1 is likely not only to spare these cells enabling re-epithelialization, but also to manage chronic inflammation caused by release of this DAMP molecule. Additional studies testing these multifaceted effects of SPD in a chronic wound setting are thus warranted.

\section{Materials and methods}

Surfactant polymer dressing. PluroGel (Medline Industries, Inc. Northfield, IL), which contains Poloxamer 188, was employed as SPD in all the experiments. SPD was reconstituted to $1 \mathrm{~g} / \mathrm{ml}$ in Dulbecco's phosphate-buffered saline (DPBS, Gibco, Carlsbad, CA \# 14190250) to make the stock solution which was then filtered using $0.2 \mu \mathrm{m}$ filter. Further, it was diluted in media and used to treat the cells.

Cell culture. Immortalized human keratinocytes (HaCaT) cells were grown at $37{ }^{\circ} \mathrm{C}$ temperature in a humidified atmosphere consisting of $95 \% \mathrm{O}_{2}$ and $5 \% \mathrm{CO}_{2}$ in Dulbecco's modified Eagle's medium (DMEM, low glucose, Gibco, Carlsbad, CA \# 11885092) that was supplemented with 10\% FBS (Gibco, Carlsbad, CA), 100 IU/ $\mathrm{ml}$ penicillin, and $0.1 \mathrm{mg} / \mathrm{ml}$ streptomycin ${ }^{37,95,96}$.

Primary Human Epidermal Keratinocytes (HEKa) cells (ATCC, Manassas, VA \#PCS-200-011) were cultured in dermal cell basal media (ATCC, Manassas, VA \#PCS-200-030) supplemented with human keratinocyte growth supplement (ATCC, Manassas, VA \#PCS-200-040) and Penicillin-Streptomycin-Amphotericin B solution (ATCC, Manassas, VA \#PCS-999-002) and incubated at $37^{\circ} \mathrm{C}$ in a $\mathrm{CO}_{2}$ incubator. After every $48 \mathrm{~h}$, the media was changed until the cells reached $80-90 \%$ confluency. HEKa cells would start to become senescent at about passage 3 or 4 . Therefore, all the experiments were performed with cells at passages 2 to prevent cell senescence.

Characterization of cell death by flow cytometry. Cells were grown in twelve-well plates at a seeding density of $0.1 \mathrm{million} / \mathrm{ml}$. After reaching confluence, typically after $24 \mathrm{~h}$, cells were treated with TX100 with and without SPD. Cell viability was measured using propidium iodide staining as previously described ${ }^{97-99}$. After 
A

DAPI

HMGB1

merged

co-localization
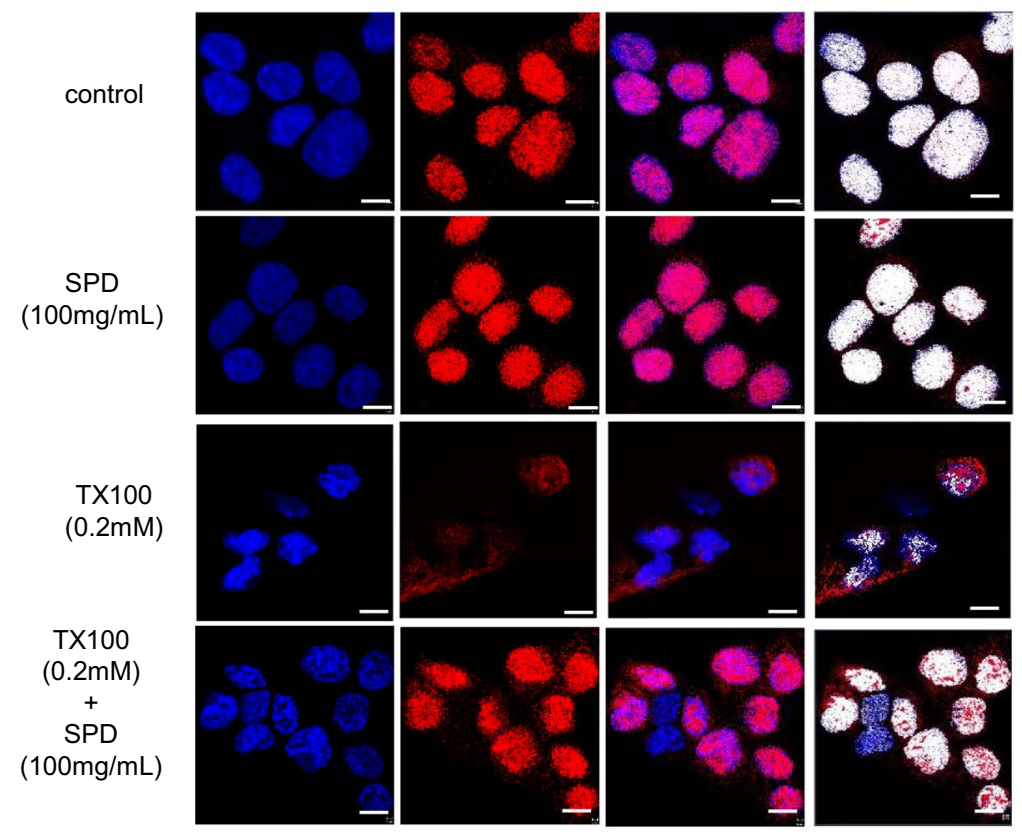

B

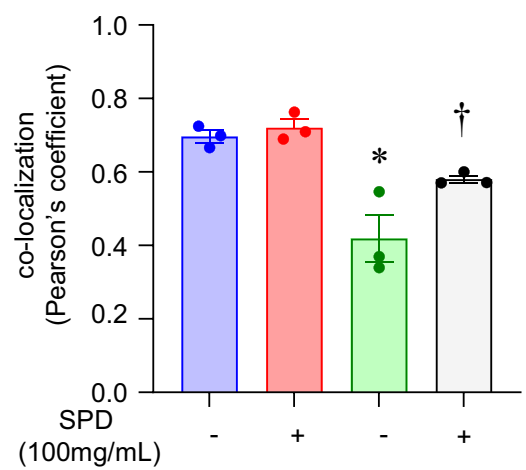

TX100

(0.2mM)

D

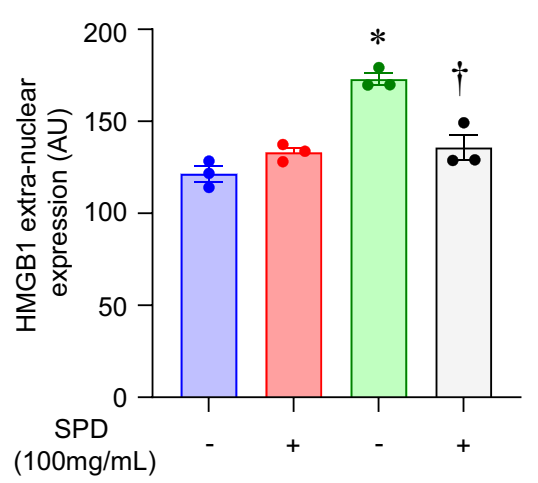

TX100

(0.2mM)
C

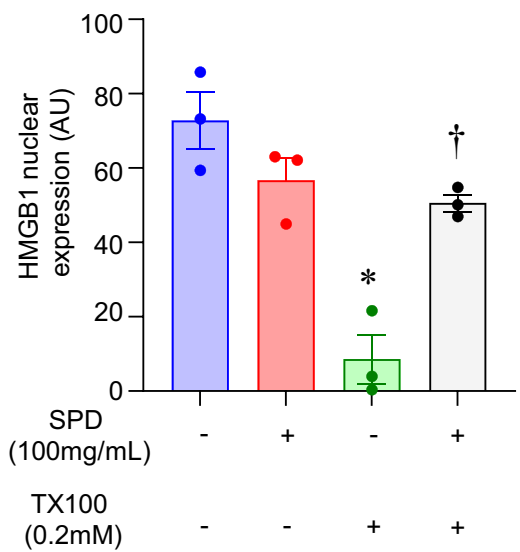

E

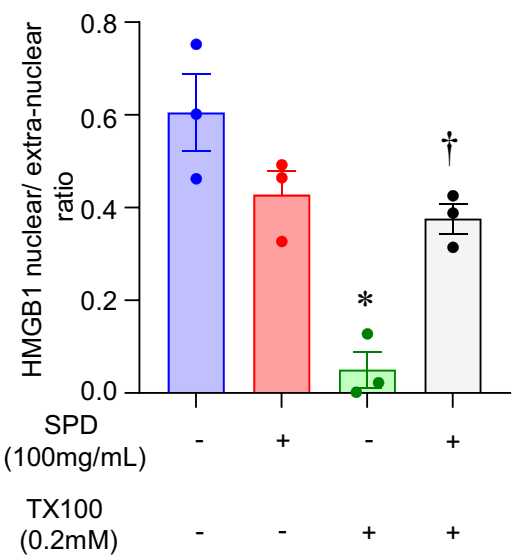


4 Figure 5. SPD rescued TX100-induced nuclear depletion of HMGB1. Human keratinocytes (HaCaT) were exposed to TX100 $(0.2 \mathrm{mM})$ for $3 \mathrm{~h}$ in presence of SPD $(100 \mathrm{mg} / \mathrm{ml})$. Cells were then fixed and subjected to immunocytochemistry using HMGB1 antibody and DAPI as nuclear stain (A) Representative microscopic images of HMGB1 (red) and DAPI (blue) as assessed by immunocytochemistry in combination with confocal microscopy. Co-localization was performed using Zen Blue software (v.3.1). Scale, $10 \mu \mathrm{m}$. (B) Co-localization of DAPI and HMGB1 as assessed by Pearson's coefficient. Data represent mean \pm SEM. $(n=3) .{ }^{*} p<0.05$ compared to control (TX100-untreated). ${ }^{\dagger} p<0.05$ compared to TX100-treated group. (C,D) Quantification of nuclear and extranuclear HMGB1 was analyzed by Zen Blue software (v.3.1). Data represent mean \pm SEM. $(n=3)$. ${ }^{*} p<0.05$ compared to control (TX100-untreated). ${ }^{\dagger} p<0.05$ compared to TX100-treated group. (E) Ratio of nuclear to extranuclear HMGB1. Data represent mean \pm SEM. $(n=3) .{ }^{\star} p<0.05$ compared to control (TX100-untreated). ${ }^{\dagger} p<0.05$ compared to TX100-treated group.

the treatments, floating dead cells were collected from the medium. The attached cells were trypsinized using trypsin-EDTA (Gibco, Carlsbad, CA \#15400054) and then collected into the 4\% FBS. The suspension of dead cells was centrifuged at 15,000 $\mathrm{g}$ for $10 \mathrm{~min}$, while the suspension of trypsinized cells was centrifuged at $\sim 700 \mathrm{~g}$ for $10 \mathrm{~min}$. Subsequently, the pellets were mixed in a $200 \mu \mathrm{L}$ of PI $(10 \mu \mathrm{g} / \mathrm{ml}$, diluted in $4 \% \mathrm{FBS})$ and incubated at room temperature for $15 \mathrm{~min}$. The flow cytometry measurements were performed using the BD Accuri flow cytometry (BD Biosciences, USA) and analyzed as previously described ${ }^{100,101}$.

Characterization of cell death by confocal microscopy. Cells were grown in the $35 \mathrm{~mm}$ Quad $\mu$-Dish (ibidi, Gräfelfing, Germany \# 80416). After reaching confluence, typically after $24 \mathrm{~h}$, cells were treated with TX100 with and without SPD containing calcein-AM (Life Technologies, Carlsbad, CA \# C3099) and propidium iodide (Sigma-Aldrich, St. Louis, MO \# P4170). The confocal imaging was performed at $0 \mathrm{~h}$ and $3 \mathrm{~h}$.

Immunocytochemistry. Cells were grown and treated in the 8-well chamber slides (Thermo Scientific Nunc Lab-Tek, Waltham, MA, \# 12-565-22). The attached cells were washed with DPBS and were subjected to immunocytochemistry as previously described ${ }^{3,37,100,101,102}$. Subsequently, cells were fixed with intracellular (IC) fixation buffer (eBioscience, San Diego, CA, \# 00-8222-49) followed by the permeabilization using $0.1 \%$ Triton $\mathrm{X}-100^{3,37,100,101,102}$. The cells were incubated with $10 \%$ normal goat serum (NGS, Vector Labs, Inc, Burlingame, CA, \# S-1000) for $1 \mathrm{~h}$ at room temperature for blocking non-specific binding of antibodies ${ }^{3,37,100,101,102}$. Next, cells were exposed to the primary antibodies made in $2.5 \%$ NGS overnight at $4{ }^{\circ} \mathrm{C}$. Following antibodies have been used: HMGB1 antibody (Cell Signaling Technology, Inc., Danvers, MA, \# 3935S), RIP3 antibody (Cell Signaling Technology, Inc., Danvers, MA, \# 13526S), MLKL (phospho-Ser358) antibody (Biorbyt, Cambridge, UK \# orb499999). The cells were washed with PBST for 5 min (twice) followed by the incubation with a secondary antibody made in $2.5 \%$ NGS for $1 \mathrm{~h}$ at room temperature in the dark $3,37,100,101,102$. The cells were again washed with PBST, followed by the staining with DAPI (Thermo Scientific, Waltham, MA \# 62248) and mounted with mounting media (Vector Labs, Inc, Burlingame, CA \# H-1400) 3,37,100,101,102. The microscopic images were captured with a Zeiss LSM 880 Airyscan super-resolution confocal microscope ${ }^{100}$. For mitochondrial staining, the cells were incubated with MitoTrackerRedCMXRos (Invitrogen, Carlsbad, CA \# M7512) for 30 min before fixation. Intensity and Pearson's coefficient were analyzed using ImageJ and Zen Blue software (v.3.1), respectively ${ }^{96,100}$. The nuclear and extranuclear localization of HMGB1 was quantified using Zen Blue software (v.3.1). Briefly, a colocalized image of HMGB1 with DAPI was extracted by masking the extranuclear HMGB1 (no colocalized with DAPI. Extranuclear HMGB1 was quantified by the following formula:

Extranuclear HMGB1 intensity = Total HMGB1 intensity-masked HMGB1 intensity.

Statistical analyses. All the data are reported as mean \pm SEM of three to nine experiments, as indicated in respective figure legends. One-way ANOVA was used to determine significant differences. A $p$ value $<0.05$ was considered statistically significant. 


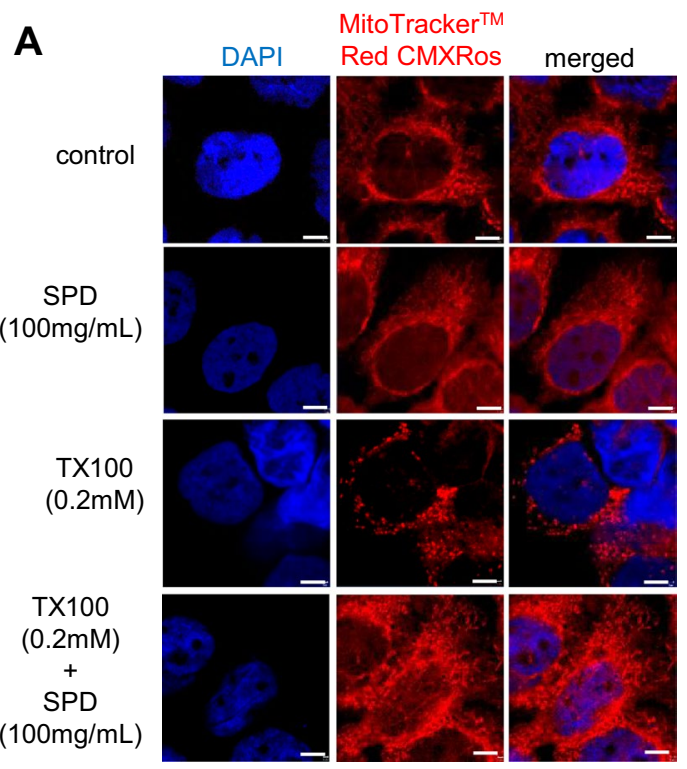

\section{B}

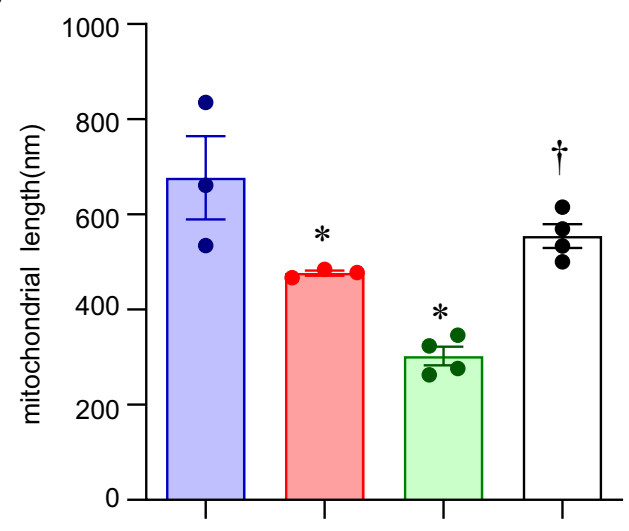

SPD

$(100 \mathrm{mg} / \mathrm{mL})$

TX100

$(0.2 \mathrm{mM})$

Figure 6. SPD rescued TX100-induced mitochondrial fragmentation. Human keratinocytes (HaCaT) were exposed to TX100 $(0.2 \mathrm{mM})$ for $1 \mathrm{~h}$ in presence of SPD $(100 \mathrm{mg} / \mathrm{ml})$. Cells were then stained with MitoTrackerRedCMXRos. (A,B) Representative microscopic images and quantification of tubular length of the mitochondria was assessed by confocal microscopy for at least 100 mitochondria selected from the three different regions of interests per sample. Scale, $10 \mu \mathrm{m}$. Data represent mean \pm SEM $(n=3-4) .{ }^{*} p<0.05$ compared to control (TX100-untreated). ${ }^{\dagger} p<0.05$ compared to TX100-treated group. 
A



RIP3

merged

B

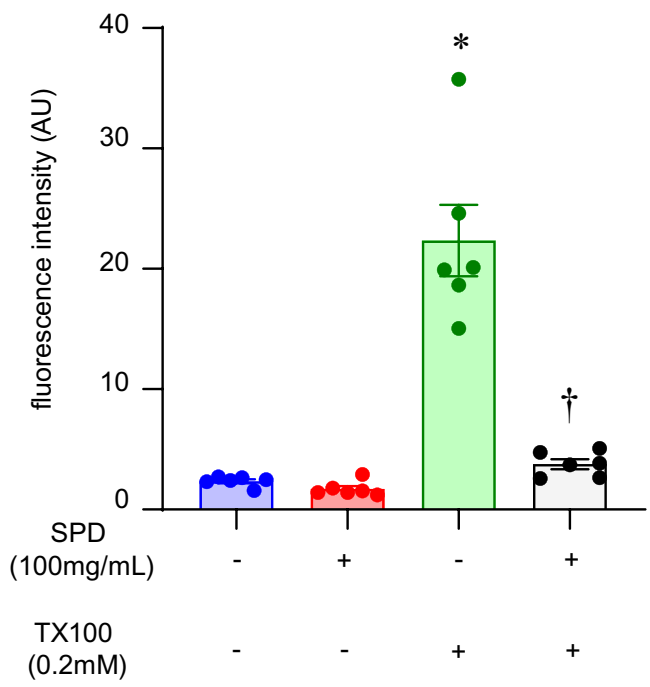

Figure 7. SPD blunted TX100-induced RIP3 expression. Human keratinocytes (HaCaT) were exposed to TX100 $(0.2 \mathrm{mM})$ for $3 \mathrm{~h}$ in presence of SPD $(100 \mathrm{mg} / \mathrm{ml})$. Cells were then fixed and immunostained with RIP3 antibody. (A,B) Representative microscopic images and quantification of RIP3 expression was assessed by confocal microscopy. Scale, $10 \mu \mathrm{m}$. Data represent mean \pm SEM $(n=6) .{ }^{*} p<0.05$ compared to control (TX100untreated). ${ }^{\dagger} p<0.05$ compared to TX100-treated group. 
A

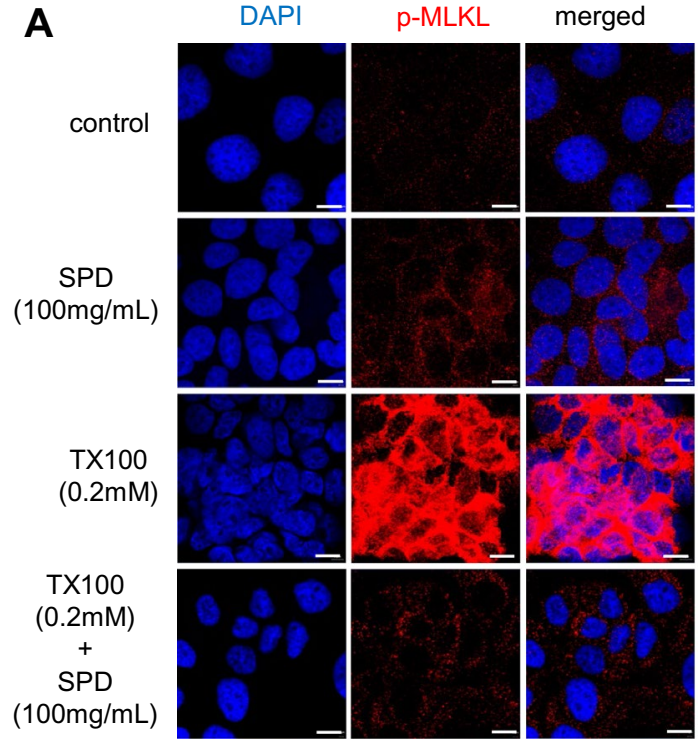

merged

B

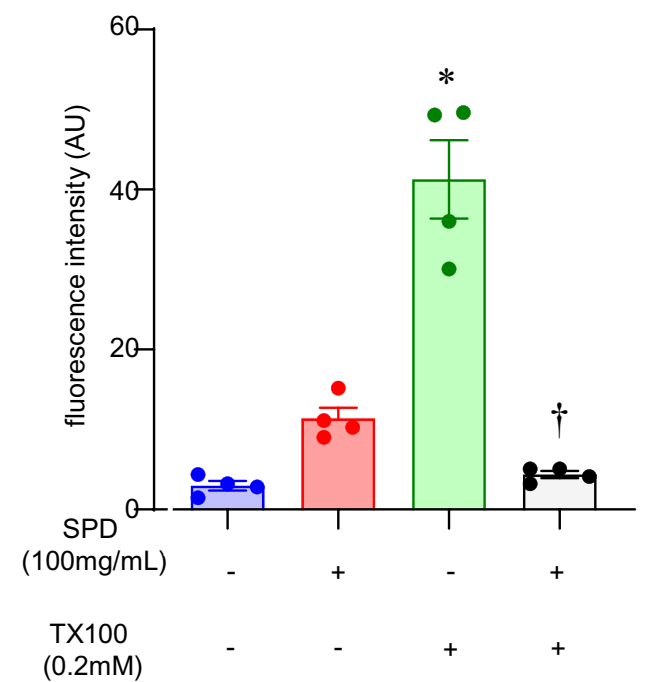

Figure 8. SPD blunted TX100-induced phosphorylation of MLKL. Human keratinocytes (HaCaT) were exposed to TX100 $(0.2 \mathrm{mM})$ for $3 \mathrm{~h}$ in presence of SPD $(100 \mathrm{mg} / \mathrm{ml})$. Cells were then fixed and immunostained with phospho-MLKL antibody. (A,B) Representative microscopic images and quantification of phospho-MLKL was assessed by confocal microscopy. Scale, $10 \mu \mathrm{m}$. Data represent mean $\pm \operatorname{SEM}(n=4) .{ }^{*} p<0.05$ compared to control (TX100-untreated). ${ }^{\dagger} p<0.05$ compared to TX100-treated group.

\section{Data availability}

All data generated or analyzed during this study are included in this published article (and its Supplementary Information files).

Received: 12 June 2020; Accepted: 27 October 2020

Published online: 23 February 2021

\section{References}

1. Broszczak, D. A., Sydes, E. R., Wallace, D. \& Parker, T. J. Molecular aspects of wound healing and the rise of venous leg ulceration: omics approaches to enhance knowledge and aid diagnostic discovery. Clin. Biochem. Rev. 38, 35-55 (2017).

2. Khanna, S. et al. Macrophage dysfunction impairs resolution of inflammation in the wounds of diabetic mice. PLoS ONE $\mathbf{5}$, e9539 (2010).

3. Das, A. et al. Correction of MFG-E8 resolves inflammation and promotes cutaneous wound healing in diabetes. J. Immunol. 196, 5089-5100 (2016).

4. Das, A., Ganesh, K., Khanna, S., Sen, C. K. \& Roy, S. Engulfment of apoptotic cells by macrophages: a role of microRNA-21 in the resolution of wound inflammation. J. Immunol. 192, 1120-1129 (2014).

5. Sen, C. K. Wound healing essentials: let there be oxygen. Wound Repair Regen. 17, 1-18 (2009). 
6. Wiegering, A. et al. Impact of incisional negative pressure wound therapy on perineal wound healing after abdominoperineal rectum extirpation. Int. J. Colorectal Dis. 32, 291-293 (2017).

7. Dryburgh, N., Smith, F., Donaldson, J. \& Mitchell, M. Debridement for surgical wounds. Cochrane Database Syst. Rev., CD006214 (2008).

8. Halim, A. S., Khoo, T. L. \& Saad, A. Z. Wound bed preparation from a clinical perspective. Indian J. Plast. Surg. 45, 193-202 (2012).

9. Jones, V., Grey, J. E. \& Harding, K. G. Wound dressings. BMJ 332, 777-780 (2006).

10. Turner, N. J. \& Badylak, S. F. The use of biologic scaffolds in the treatment of chronic nonhealing wounds. Adv. Wound Care (New Rochelle) 4, 490-500 (2015).

11. Dabiri, G., Damstetter, E. \& Phillips, T. Choosing a wound dressing based on common wound characteristics. Adv. Wound Care (New Rochelle) 5, 32-41 (2016).

12. Pastar, I. et al. Epithelialization in wound healing: a comprehensive review. Adv. Wound Care (New Rochelle) 3, 445-464 (2014).

13. Husmann, M. et al. Elimination of a bacterial pore-forming toxin by sequential endocytosis and exocytosis. FEBS Lett. 583, 337-344 (2009).

14. Gonzalez, M. R. et al. Pore-forming toxins induce multiple cellular responses promoting survival. Cell. Microbiol. 13, 1026-1043 (2011).

15. Tam, C., Flannery, A. R. \& Andrews, N. Live imaging assay for assessing the roles of $\mathrm{Ca}^{2+}$ and sphingomyelinase in the repair of pore-forming toxin wounds. J. Vis. Exp. 78, e50531 (2013).

16. LaRocca, T. J. et al. Human-specific bacterial pore-forming toxins induce programmed necrosis in erythrocytes. $m$ Bio 5 , e0125101214 (2014).

17. Gonzalez-Juarbe, N. et al. Pore-forming toxins induce macrophage necroptosis during acute bacterial pneumonia. PLoS Pathog. 11, e1005337 (2015).

18. Cabezas, S. et al. Damage of eukaryotic cells by the pore-forming toxin sticholysin II: Consequences of the potassium efflux. Biochim. Biophys. Acta Biomembr. 1859, 982-992 (2017).

19. Gonzalez-Juarbe, N. et al. Bacterial pore-forming toxins promote the activation of caspases in parallel to necroptosis to enhance alarmin release and inflammation during pneumonia. Sci. Rep. 8, 5846 (2018).

20. Brito, C., Cabanes, D., Sarmento Mesquita, F. \& Sousa, S. Mechanisms protecting host cells against bacterial pore-forming toxins. Cell Mol. Life Sci. 76, 1319-1339 (2019).

21. Larpin, Y. et al. Bacterial pore-forming toxin pneumolysin: cell membrane structure and microvesicle shedding capacity determines differential survival of cell types. FASEB J. 34, 1665-1678 (2020).

22. Andrade, L. O. Plasma membrane repair involvement in parasitic and other pathogen infections. Curr. Top. Membr. 84, 217-238 (2019).

23. Cao, J. Y. \& Dixon, S. J. Mechanisms of ferroptosis. Cell Mol. Life Sci. 73, 2195-2209 (2016).

24. Liang, H. \& Liu, Y. Gasdermins pore cell membrane to pyroptosis. Sci. China Life Sci. 59, 1090-1092 (2016).

25. Kovacs, S. B. \& Miao, E. A. Gasdermins: effectors of pyroptosis. Trends Cell Biol. 27, 673-684 (2017).

26. McKenzie, B. A., Dixit, V. M. \& Power, C. Fiery cell death: pyroptosis in the central nervous system. Trends Neurosci. 43, 55-73 (2020).

27. Kaczmarek, A., Vandenabeele, P. \& Krysko, D. V. Necroptosis: the release of damage-associated molecular patterns and its physiological relevance. Immunity 38, 209-223 (2013).

28. Cai, Z. et al. Plasma membrane translocation of trimerized MLKL protein is required for TNF-induced necroptosis. Nat. Cell Biol. 16, 55-65 (2014).

29. Ros, U. et al. Necroptosis execution is mediated by plasma membrane nanopores independent of calcium. Cell Rep. 19, 175-187 (2017).

30. Yang, S., Xu, M., Meng, G. \& Lu, Y. SIRT3 deficiency delays diabetic skin wound healing via oxidative stress and necroptosis enhancement. J. Cell. Mol. Med. 24, 4415-4427 (2020).

31. Wang, H. et al. Mixed lineage kinase domain-like protein MLKL causes necrotic membrane disruption upon phosphorylation by RIP3. Mol. Cell 54, 133-146 (2014).

32. Chen, X. et al. Translocation of mixed lineage kinase domain-like protein to plasma membrane leads to necrotic cell death. Cell Res. 24, 105-121 (2014).

33. Kwiatkowski, T. A. et al. Multiple poloxamers increase plasma membrane repair capacity in muscle and non-muscle cells. Am. J. Physiol. Cell Physiol. 318, C253-C262 (2019).

34. Moloughney, J. G. \& Weisleder, N. Poloxamer 188 (p188) as a membrane resealing reagent in biomedical applications. Recent Pat. Biotechnol. 6, 200-211 (2012).

35. Das Ghatak, P., Mathew-Steiner, S. S., Pandey, P., Roy, S. \& Sen, C. K. A surfactant polymer dressing potentiates antimicrobial efficacy in biofilm disruption. Sci. Rep. 8, 873 (2018).

36. Roy, S. et al. Mixed-species biofilm compromises wound healing by disrupting epidermal barrier function. J. Pathol. 233, 331-343 (2014).

37. Barki, K. G. et al. Electric field based dressing disrupts mixed-species bacterial biofilm infection and restores functional wound healing. Ann. Surg. 269, 756-766 (2019).

38. Singhal, A., Reis, E. D. \& Kerstein, M. D. Options for nonsurgical debridement of necrotic wounds. Adv. Skin Wound Care 14, 96-100 (2001) ((quiz 102-103)).

39. Larouche, J., Sheoran, S., Maruyama, K. \& Martino, M. M. Immune regulation of skin wound healing: mechanisms and novel therapeutic targets. Adv. Wound Care (New Rochelle) 7, 209-231 (2018).

40. Eming, S. A., Krieg, T. \& Davidson, J. M. Inflammation in wound repair: molecular and cellular mechanisms. J. Invest. Dermatol. $127,514-525$ (2007)

41. Beckwith, K. S. et al. Plasma membrane damage causes NLRP3 activation and pyroptosis during Mycobacterium tuberculosis infection. Nat. Commun. 11, 2270 (2020).

42. de Armas-Rillo, L., Valera, M. S., Marrero-Hernandez, S. \& Valenzuela-Fernandez, A. Membrane dynamics associated with viral infection. Rev. Med. Virol. 26, 146-160 (2016).

43. Rock, K. L. \& Kono, H. The inflammatory response to cell death. Annu. Rev. Pathol. 3, 99-126 (2008).

44. Lange, S. S., Mitchell, D. L. \& Vasquez, K. M. High mobility group protein B1 enhances DNA repair and chromatin modification after DNA damage. Proc. Natl. Acad. Sci. USA 105, 10320-10325 (2008).

45. Lange, S. S. \& Vasquez, K. M. HMGB1: the jack-of-all-trades protein is a master DNA repair mechanic. Mol. Carcinog. 48, 571-580 (2009).

46. Liu, Y. et al. Extracellular HMGB1 prevents necroptosis in acute myeloid leukemia cells. Biomed. Pharmacother. 112, 108714 (2019).

47. Tang, D. et al. High-mobility group box 1 is essential for mitochondrial quality control. Cell Metab. 13, 701-711 (2011).

48. Borner, M. M. et al. The detergent Triton X-100 induces a death pattern in human carcinoma cell lines that resembles cytotoxic lymphocyte-induced apoptosis. FEBS Lett. 353, 129-132 (1994).

49. Sawai, H. \& Domae, N. Release of cytochrome c from mitochondria precedes Bax translocation/activation in Triton X-100-induced apoptosis. Leuk. Res. 32, 445-453 (2008). 
50. Sawai, H. \& Domae, N. Differential roles for Bak in Triton X-100- and deoxycholate-induced apoptosis. Biochem. Biophys. Res. Commun. 378, 529-533 (2009).

51. Cambiaso-Daniel, J. et al. Topical antimicrobials in burn care: part 1-topical antiseptics. Ann. Plast. Surg. https://doi. org/10.1097/SAP.0000000000001297 (2018).

52. FDA. https://www.accessdata.fda.gov/scripts/cdrh/cfdocs/cfrl/ldetails.cfm?lid=184268.

53. Kirsner, R. S. et al. Effects of a surfactant-based gel on acute and chronic paediatric wounds: a panel discussion and case series. J. Wound Care 28, 398-408 (2019).

54. Woo, K. et al. Effect of a surfactant-based gel on patient quality of life. J. Wound Care 27, 664-678 (2018).

55. Palumbo, F. P. et al. New surfactant-based dressing product to improve wound closure rates of nonhealing wounds: a European multicenter study including 1036 patients. Wounds 28, 233-240 (2016).

56. Bonnet, M. C. et al. The adaptor protein FADD protects epidermal keratinocytes from necroptosis in vivo and prevents skin inflammation. Immunity 35, 572-582 (2011).

57. Horn, A. \& Jaiswal, J. K. Cellular mechanisms and signals that coordinate plasma membrane repair. Cell. Mol. Life Sci. 75, 3751-3770 (2018).

58. Andrews, N. W. \& Corrotte, M. Plasma membrane repair. Curr. Biol. 28, R392-R397 (2018).

59. Blazek, A. D., Paleo, B. J. \& Weisleder, N. Plasma membrane repair: a central process for maintaining cellular homeostasis. Physiology 30, 438-448 (2015).

60. Andrews, N. W. \& Perez, F. The plasma membrane repair shop: Fixing the damage. Semin. Cell Dev. Biol. 45, 1 (2015).

61. Draeger, A., Schoenauer, R., Atanassoff, A. P., Wolfmeier, H. \& Babiychuk, E. B. Dealing with damage: plasma membrane repair mechanisms. Biochimie 107(Pt A), 66-72 (2014).

62. Pathak-Sharma, S., Zhang, X., Lam, J. G. T., Weisleder, N. \& Seveau, S. M. High-throughput microplate-based assay to monitor plasma membrane wounding and repair. Front. Cell Infect. Microbiol. 7, 305 (2017).

63. Jimenez, A. J., Maiuri, P., Lafaurie-Janvore, J., Perez, F. \& Piel, M. Laser induced wounding of the plasma membrane and methods to study the repair process. Methods Cell Biol. 125, 391-408 (2015).

64. Bashford, C. L. et al. Membrane damage by hemolytic viruses, toxins, complement, and other cytotoxic agents. A common mechanism blocked by divalent cations. J. Biol. Chem. 261, 9300-9308 (1986).

65. Koley, D. \& Bard, A. J. Triton X-100 concentration effects on membrane permeability of a single HeLa cell by scanning electrochemical microscopy (SECM). Proc. Natl. Acad. Sci. USA 107, 16783-16787 (2010).

66. Rusanov, A. L. et al. Changes in the proteome of HaCaT keratinocytes induced by cytotoxic substance Triton X-100. Bull. Exp. Biol. Med. 163, 620-622 (2017).

67. Strupp, W. et al. Treatment of cells with detergent activates caspases and induces apoptotic cell death. J. Membr. Biol. 175, 181-189 (2000).

68. Nyholm, T. \& Slotte, J. P. Comparison of Triton X-100 penetration into phosphatidylcholine and sphingomyelin mono- and bilayers. Langmuir 17, 4724-4730 (2001).

69. le Maire, M., Champeil, P. \& Moller, J. V. Interaction of membrane proteins and lipids with solubilizing detergents. Biochim. Biophys. Acta 1508, 86-111 (2000).

70. Simonetti, O. et al. Plasma membrane fluidity of keratinocytes of normal and psoriatic skin: a study using fluorescence anisotropy of trimethylammoniumdiphenylhexatriene (TMA-DPH). Arch. Dermatol. Res. 288, 51-54 (1996).

71. van de Ven, A. L., Adler-Storthz, K. \& Richards-Kortum, R. Delivery of optical contrast agents using Triton-X100, part 1: reversible permeabilization of live cells for intracellular labeling. J. Biomed. Opt. 14, 021012 (2009).

72. Bogner, P., Kellermayer, M. \& Hazlewood, C. F. Release of hemoglobin and potassium from human red blood cells treated with Triton X-100 under the critical micellar concentration. Scanning Microsc. 3, 1241-1243 (1989) ((discussion 1244-1245)).

73. van Smeden, J., Janssens, M., Gooris, G. S. \& Bouwstra, J. A. The important role of stratum corneum lipids for the cutaneous barrier function. Biochim. Biophys. Acta 1841, 295-313 (2014).

74. Henseleit, U., Rosenbach, T. \& Kolde, G. Induction of apoptosis in human HaCaT keratinocytes. Arch. Dermatol. Res. 288, 676-683 (1996).

75. Cochilla, A. J., Angleson, J. K. \& Betz, W. J. Monitoring secretory membrane with FM1-43 fluorescence. Annu. Rev. Neurosci. 22, 1-10 (1999).

76. Li, X. et al. Cell membrane damage is involved in the impaired survival of bone marrow stem cells by oxidized low-density lipoprotein. J. Cell. Mol. Med. 18, 2445-2453 (2014).

77. Rayamajhi, M. \& Miao, E. A. The RIP1-RIP3 complex initiates mitochondrial fission to fuel NLRP3. Nat. Immunol. 15, 1100-1102 (2014).

78. Scott, I. \& Youle, R. J. Mitochondrial fission and fusion. Essays Biochem. 47, 85-98 (2010).

79. Shen, Y. L. et al. TNF-alpha induces Drp1-mediated mitochondrial fragmentation during inflammatory cardiomyocyte injury. Int. J. Mol. Med. 41, 2317-2327 (2018).

80. Jogdand, P. S. et al. Flow cytometric readout based on Mitotracker Red CMXRos staining of live asexual blood stage malarial parasites reliably assesses antibody dependent cellular inhibition. Malar. J. 11, 235 (2012).

81. Kim, J. E. \& Kang, T. C. Differential roles of mitochondrial translocation of active caspase-3 and HMGB1 in neuronal death induced by status epilepticus. Front. Cell. Neurosci. 12, 301 (2018).

82. Huebener, P. et al. High-mobility group box 1 is dispensable for autophagy, mitochondrial quality control, and organ function in vivo. Cell Metab. 19, 539-547 (2014).

83. Raucci, A., Palumbo, R. \& Bianchi, M. E. HMGB1: a signal of necrosis. Autoimmunity 40, 285-289 (2007)

84. Nogueira-Machado, J. A., Volpe, C. M., Veloso, C. A. \& Chaves, M. M. HMGB1, TLR and RAGE: a functional tripod that leads to diabetic inflammation. Expert Opin. Ther. Targets 15, 1023-1035 (2011).

85. Yu, M. et al. HMGB1 signals through toll-like receptor (TLR) 4 and TLR2. Shock 26, 174-179 (2006).

86. Apetoh, L. et al. The interaction between HMGB1 and TLR4 dictates the outcome of anticancer chemotherapy and radiotherapy. Immunol. Rev. 220, 47-59 (2007).

87. Weber, K., Roelandt, R., Bruggeman, I., Estornes, Y. \& Vandenabeele, P. Nuclear RIPK3 and MLKL contribute to cytosolic necrosome formation and necroptosis. Commun. Biol. 1, 6 (2018).

88. Wu, X. N. et al. Distinct roles of RIP1-RIP3 hetero- and RIP3-RIP3 homo-interaction in mediating necroptosis. Cell Death Differ. 21, 1709-1720 (2014).

89. Yang, Z. et al. RIP3 targets pyruvate dehydrogenase complex to increase aerobic respiration in TNF-induced necroptosis. Nat. Cell Biol. 20, 186-197 (2018).

90. Zhang, L., Feng, Q. \& Wang, T. Necrostatin-1 protects against paraquat-induced cardiac contractile dysfunction via RIP1-RIP3MLKL-dependent necroptosis pathway. Cardiovasc. Toxicol. 18, 346-355 (2018).

91. Dondelinger, Y. et al. MLKL compromises plasma membrane integrity by binding to phosphatidylinositol phosphates. Cell Rep. 7, 971-981 (2014).

92. Murphy, J. M. The killer pseudokinase mixed lineage kinase domain-like protein (MLKL). Cold Spring Harb. Perspect. Biol. 12, a036376 (2019).

93. Conos, S. A. et al. Active MLKL triggers the NLRP3 inflammasome in a cell-intrinsic manner. Proc. Natl. Acad. Sci. USA 114, E961-E969 (2017). 
94. Duan, X. et al. Inhibition of keratinocyte necroptosis mediated by RIPK1/RIPK3/MLKL provides a protective effect against psoriatic inflammation. Cell Death Dis. 11, 134 (2020).

95. Biswas, S. et al. Hypoxia inducible microRNA 210 attenuates keratinocyte proliferation and impairs closure in a murine model of ischemic wounds. Proc. Natl. Acad. Sci. USA 107, 6976-6981 (2010).

96. Singh, K. et al. Cutaneous epithelial to mesenchymal transition activator ZEB1 regulates wound angiogenesis and closure in a glycemic status-dependent manner. Diabetes 68, 2175-2190 (2019).

97. Khanna, S. et al. Loss of miR-29b following acute ischemic stroke contributes to neural cell death and infarct size. J. Cereb. Blood Flow Metab. 33, 1197-1206 (2013).

98. Sen, C. K., Khanna, S., Roy, S. \& Packer, L. Molecular basis of vitamin E action. Tocotrienol potently inhibits glutamate-induced pp60(c-Src) kinase activation and death of HT4 neuronal cells. J. Biol. Chem. 275, 13049-13055 (2000).

99. Khanna, S. et al. Molecular basis of vitamin E action: tocotrienol modulates 12-lipoxygenase, a key mediator of glutamateinduced neurodegeneration. J. Biol. Chem. 278, 43508-43515 (2003).

100. Das, A. et al. A modified collagen dressing induces transition of inflammatory to reparative phenotype of wound macrophages. Sci. Rep. 9, 14293 (2019).

101. Das, A. et al. Novel mechanisms of Collagenase Santyl Ointment (CSO) in wound macrophage polarization and resolution of wound inflammation. Sci. Rep. 8, 1696 (2018).

102. Ghosh, N. et al. Urolithin A augments angiogenic pathways in skeletal muscle by bolstering NAD+ and SIRT1. Sci. Rep. 10, 20184 (2020).

\section{Acknowledgements}

Human immortalized keratinocytes (HaCaT) was provided kindly by Dr. NE Fusenig of German Cancer Research Center, Heidelberg, Germany. This work was supported by the National Institutes of Health Grants U01DK119099 and R01GM108014 to CKS, R01NR015676 to CKS and SR and R01DK114718 to SR.

\section{Author contributions}

S.K., A.D., C.K.S. and P.K. conceived and designed the work. P.K., A.D., C.K.S., S.P.S., S.R. and S.K. collected, analyzed data for this work and participated in the preparation of the manuscript S.K., A.D., C.K.S. and P.K. wrote the manuscript. All authors reviewed the manuscript.

\section{Competing interests}

The authors declare that SPD and partial research funding was provided by Medline Industries Inc. No authors have any conflict of interest.

\section{Additional information}

Supplementary Information The online version contains supplementary material available at https://doi. org/10.1038/s41598-021-82260-x.

Correspondence and requests for materials should be addressed to S.K.

Reprints and permissions information is available at www.nature.com/reprints.

Publisher's note Springer Nature remains neutral with regard to jurisdictional claims in published maps and institutional affiliations.

(c) (i) Open Access This article is licensed under a Creative Commons Attribution 4.0 International License, which permits use, sharing, adaptation, distribution and reproduction in any medium or format, as long as you give appropriate credit to the original author(s) and the source, provide a link to the Creative Commons licence, and indicate if changes were made. The images or other third party material in this article are included in the article's Creative Commons licence, unless indicated otherwise in a credit line to the material. If material is not included in the article's Creative Commons licence and your intended use is not permitted by statutory regulation or exceeds the permitted use, you will need to obtain permission directly from the copyright holder. To view a copy of this licence, visit http://creativecommons.org/licenses/by/4.0/.

(c) The Author(s) 2021 